\title{
Quantum Tunneling of the Non-stationary Kerr-Newman Black Hole via a New Type of General Tortoise Coordinate Transformation
}

\author{
Zhong-Wen Feng • Juan Deng • Guo-Ping Li • \\ Shu-Zheng Yang
}

Received: 26 November 2011 / Accepted: 7 May 2012 / Published online: 22 May 2012

(C) The Author(s) 2012. This article is published with open access at Springerlink.com

\begin{abstract}
In this paper, the quantum tunneling of the non-stationary Kerr-Newman black hole is investigated via Hamilton-Jacobi equation and two types of general tortoise coordinate transformations. The tunneling rates, the Hawking temperatures and radiation spectrums are derived respectively. Our result shows that the new type of general tortoise coordinate transformation is more reasonable.
\end{abstract}

Keywords Non-stationary Kerr-Newman black hole · Quantum tunneling · General tortoise coordination transformation

\section{Introduction}

In 1974, Hawking proved black holes can emit thermal radiation by the view of quantum theory [1]. Subsequently, the thermodynamic property of the black holes has attracted much interest. Apart from original derivation of Hawking radiation, there are several derivations [2-5]. In 2000, Parikh and Wilczek proposed a method to study quantum tunneling. According to Palnevé coordinate transformations and WKB approach, the tunneling rate of particles from inside to outside of horizon can be written as $\Gamma \propto \exp (-2 \operatorname{Im} I)$ [6]. Along with this method, people have studied thermodynamic property of stationary black holes [7-11]. After that, Padmanabhan and collaborators proposed Hamilton-Jacobi method to study the tunneling radiation of scalar particles [12]. In 2007, Kerner and Mann proposed a new method to study fermions tunneling radiation of static black holes and Kerr-Newman black hole. With this method, fermions tunneling behavior of charged black holes, de Sitter space-time and BTZ black holes have been investigated [13-15]. The case of higherdimensional space-time and Finsler black holes is referred in [16-18], which shows that the quantum tunneling is a helpful method for us to understand the origin of Hawking radiation.

Z.-W. Feng $(\bowtie) \cdot$ J. Deng · G.-P. Li · S.-Z. Yang Institute of Theoretical Physics, China West Normal University, Nan Chong 637009, China e-mail: von198698@yahoo.com.cn 
In previous works, static and stationary black holes have been researched extensively. However, the real black holes in the universe are dynamic. Therefore, the research on nonstationary black holes has substantial significance. It is well known that the horizon of nonstationary black holes varies with the time, so, there are some problems when studying the Hawking radiation of non-stationary black holes. To solve these problems, people usually introduce the general tortoise coordinate transformation combining with the method of Damour-Ruffini. Zhao and Yang et al. which has fruitful researched on several nonstationary black holes. Recently, Yang has proposed a new type of general tortoise coordinate transformation [19] which is considered with more physically significance and benefits to study Hawking radiation.

In this article, the Hamilton-Jacobi equation was being derived from Klein-Gordon equation then the tunneling radiation of non-stationary Kerr-Newman black hole was studied via the new type of general tortoise coordinate. Comparing to the results on the previous general tortoise coordinate, it is find that the surface gravity, radiation spectrum and Hawking temperature we got are more precise.

The rest is organized as follows. In Sect. 2, we review the non-stationary Kerr-Newman black hole. In Sect. 3, we investigate the tunneling radiation of the non-stationary KerrNewman black hole under the new type of general tortoise coordinate transformation. Conclusions and discussions are devoted to Sect. 4.

\section{Non-stationary Kerr-Newman Black Hole}

The line element of the non-stationary Kerr-Newman black hole in the advanced EddingtonFinkelstein system can be written as [20-23]

$$
\begin{aligned}
\mathrm{d} s^{2}= & \frac{\Delta-a^{2} \sin ^{2} \theta}{\Sigma} \mathrm{d} v^{2}+2 \frac{r^{2}+a^{2}-\Delta}{\Sigma} a \sin ^{2} \theta \mathrm{d} v \mathrm{~d} \phi-2 \mathrm{~d} v \mathrm{~d} r \\
& +2 a \sin ^{2} \theta \mathrm{d} r \mathrm{~d} \phi-\Sigma \mathrm{d} \theta^{2}-\frac{\left[\left(r^{2}+a^{2}\right)^{2}-\Delta a^{2} \sin ^{2} \theta\right] \sin ^{2} \theta}{\Sigma} \mathrm{d} \phi^{2},
\end{aligned}
$$

with

$$
\Sigma=r^{2}+a^{2} \cos ^{2} \theta, \quad \Delta=r^{2}-2 M r+a^{2}+Q^{2},
$$

where $Q$ and $M$ are the charge and mass of the black hole, they are functions depending on coordinate $v$. The gauge potential is

$$
A_{\mu}=\frac{Q r}{\Sigma}\left(d v-a \sin ^{2} \theta d \phi\right)
$$

The determinant and the component of inverse tensors are

$$
\begin{gathered}
g=-\Sigma^{2} \sin ^{2} \theta, \\
g^{01}=g^{10}=-\frac{r^{2}+a^{2}}{\Sigma}, \quad g^{00}=-\frac{a^{2} \sin ^{2} \theta}{\Sigma}, \quad g^{11}=-\frac{\Delta}{\Sigma}, \\
g^{22}=-\frac{1}{\Sigma}, \quad g^{33}=-\frac{1}{\Sigma \sin ^{2} \theta}, \quad g^{03}=g^{30}=-\frac{a}{\Sigma}, \\
g^{13}=g^{31}=-\frac{a}{\Sigma},
\end{gathered}
$$


Generally speaking, black holes exits three types of horizons, they are Apparent horizon $(\mathrm{AH})$, the Time-like surface and Event horizon $(\mathrm{EH})$, for non-stationary black holes, the three types of horizons are not coincide. As we know, the Hawking radiation must discuss on the event horizon. The event horizon is satisfied with the null-hyper surface condition

$$
g^{\mu \nu} \frac{\partial F}{\partial x^{\mu}} \frac{\partial F}{\partial x^{\nu}}=0
$$

where $F$ means the hyper-surface. The position of event horizon is written as

$$
g^{00} \dot{r}_{H}^{2}-2 g^{01} \dot{r}_{H}+g^{11}+g^{22} r_{H}^{\prime 2}=0 .
$$

where $\dot{r}_{H}=\frac{\partial r_{H}}{\partial v}$ and $r_{H}^{\prime}=\frac{\partial r_{H}}{\partial \theta}$ describe the change of event horizon with time $v$ and angle $\theta$.

\section{Quantum Tunneling of Scalar Particles Under the New Type of General Tortoise Coordination Transformation}

The dynamic behavior of charged scalar particles in curved space-time is described by KleinGordon equation

$$
\frac{1}{\sqrt{-g}}\left(\frac{\partial}{\partial x^{\mu}}-i e A_{\mu}\right)\left[\sqrt{-g} g^{\mu \nu}\left(\frac{\partial}{\partial x^{\nu}}-i e A_{\nu}\right) \Phi\right]-\frac{m^{2}}{\hbar} \Phi=0,
$$

where $m$ and $e$ are the mass and charge of a scalar particle. The wave function $\Phi$ can been express as

$$
\Phi=\exp \left(\frac{i}{\hbar} S\right)
$$

where $S$ is the classical action. Substituting Eq. (9) into Eq. (8), the Hamilton-Jacobi equation is derived as [25]

$$
g^{\mu \nu}\left(\frac{\partial S}{\partial x^{\mu}}-e A_{\mu}\right)\left(\frac{\partial S}{\partial x^{\nu}}-e A_{v}\right)+m^{2}=0 .
$$

Equation (10) is only satisfied the scalar particles with spin 0. Putting Eqs. (4) and (5) into Eq. (10). It is get the concrete form of Hamilton-Jacobi equation

$$
\begin{aligned}
& g^{00}\left(\frac{\partial S}{\partial v}\right)^{2}+g^{11}\left(\frac{\partial S}{\partial r}\right)^{2}+g^{22}\left(\frac{\partial S}{\partial \theta}\right)^{2}+g^{33}\left(\frac{\partial S}{\partial \phi}\right)^{2}-2\left(e g^{00} A_{1}+e g^{03} A_{3}\right) \frac{\partial S}{\partial v} \\
& -2\left(g^{33} e A_{3}+g^{03} e A_{1}\right) \frac{\partial S}{\partial \phi}+2 g^{01} \frac{\partial S}{\partial v} \frac{\partial S}{\partial r}+2 g^{03} e \frac{\partial S}{\partial v} \frac{\partial S}{\partial \phi}+2 g^{13} \frac{\partial S}{\partial r} \frac{\partial S}{\partial \phi} \\
& -2 g^{01} e A_{1} \frac{\partial S}{\partial r}+g^{03} e^{2} A_{0} A_{3}-2 g^{13} e A_{3} \frac{\partial S}{\partial r}+g^{00} e^{2} A_{0}^{2}+2 g^{33} e^{2} A_{3}^{2} \\
& +m^{2}=0,
\end{aligned}
$$

For study the quantum tunneling of non-stationary black holes, the effective way is use general tortoise coordinate transformation. Now, a new type of general tortoise coordinate is defined as following [19]

$$
r_{*}=r+\alpha \ln \frac{r-r_{H}(\nu, \theta)}{r_{H}(v, \theta)},
$$




$$
\begin{aligned}
& v_{*}=v-v_{0}, \\
& \theta_{*}=\theta-\theta_{0},
\end{aligned}
$$

where $\alpha, v_{0}$ and $\theta_{0}$ are constants. According to the new type of general tortoise coordinate transformation, there are

$$
\begin{aligned}
\frac{\partial}{\partial r} & =\left[1+\frac{\alpha}{\left(r-r_{H}\right)}\right] \frac{\partial}{\partial r_{*}}, \\
\frac{\partial}{\partial v} & =\frac{\partial}{\partial \nu_{*}}-\frac{\alpha r \dot{r}_{H}}{\left(r-r_{H}\right) r_{H}} \frac{\partial}{\partial r_{*}}, \\
\frac{\partial}{\partial \theta} & =\frac{\partial}{\partial \theta_{*}}-\frac{\alpha r r_{H}^{\prime}}{\left(r-r_{H}\right) r_{H}} \frac{\partial}{\partial r_{*}} .
\end{aligned}
$$

Combining Eqs. (11)-(17), the Hamilton-Jacobi equation can be transformed into

$$
\begin{aligned}
& \frac{\left[\alpha r \dot{r}_{H}\right]^{2} a^{2} \sin ^{2} \theta+\Delta\left[\alpha+\left(r-r_{H}\right)\right]^{2} r_{H}^{2}+\left(\alpha r r_{H}^{\prime}\right)^{2}-2 a\left[\alpha+\left(r-r_{H}\right)\right] \alpha r_{H} r \dot{r}_{H}}{r_{H}\left(r-r_{H}\right)\left\{r_{H}\left(r^{2}+a^{2}\right)\left[\alpha+\left(r-r_{H}\right)\right]-\alpha r \dot{r}_{H} a^{2} \sin ^{2} \theta\right\}}\left(\frac{\partial S}{\partial r_{*}}\right)^{2} \\
& \quad+\left\{2 \frac{Q e r}{\Sigma}\left(r^{2}+a^{2}-a^{2} \sin ^{2} \theta\right)\left[\alpha+\left(r-r_{H}\right)\right] r_{H}+2 a\left[\alpha r_{H}+r_{H}\left(r-r_{H}\right)-\alpha r \dot{r}_{H}\right] j\right\} \\
& \quad \times \frac{1}{r_{H}\left(r^{2}+a^{2}\right)\left[\alpha+\left(r-r_{H}\right)\right]-\alpha r \dot{r}_{H} a^{2} \sin ^{2} \theta} \frac{\partial S}{\partial r_{*}}+2 \frac{\partial S}{\partial r_{*}} \frac{\partial S}{\partial v_{*}} \\
& -\frac{2 \alpha r r_{H}^{\prime}}{r_{H}\left(r^{2}+a^{2}\right)\left[\alpha+\left(r-r_{H}\right)\right]-\alpha r \dot{r}_{H} a^{2} \sin ^{2} \theta} \\
& \quad \times P_{\theta} \frac{\partial S}{\partial r_{*}}+\left\{\frac{e^{2} Q^{2} r^{2} \sin ^{4} \theta}{\Sigma}+3 \frac{a^{2} e^{2} Q^{2} r^{2} \sin ^{2} \theta}{\Sigma}+\Sigma m^{2}+a^{2} \sin ^{2} \theta\left(\frac{\partial S}{\partial v_{*}}\right)^{2}+\frac{j^{2}}{\sin ^{2} \theta}\right. \\
& \left.-2 \frac{Q r e}{\Sigma}\left(\frac{1}{\sin ^{2} \theta}-a^{2} \sin ^{2} \theta\right)+2 a j \frac{\partial S}{\partial v_{*}}+P_{\theta}^{2}\right\} \\
& \quad \times \frac{\left(r-r_{H}\right) r_{H}}{r_{H}\left(r^{2}+a^{2}\right)\left[\alpha+\left(r-r_{H}\right)\right]-\alpha r \dot{r}_{H} a^{2} \sin ^{2} \theta}=0,
\end{aligned}
$$

where

$$
\begin{gathered}
\mathrm{P}_{\theta}=\frac{\partial S}{\partial \theta_{*}}, \\
\mathrm{P}_{\phi}=\frac{\partial S}{\partial \phi}=j .
\end{gathered}
$$

In Eq. (20) $j$ is a constant corresponding to the Killing vector $(\partial / \partial \phi)^{\alpha}$ and related to magnetic quantum number. $\mathrm{P}_{\theta}$ is component of generalized momentum. Using the method which referred in [19], when $r$ approach the event horizon $r_{H}$, the coefficient of the term $\left(\partial R / \partial r_{*}\right)^{2}$ is $0 / 0$ form. Therefore, the limit of the coefficient can be calculated via L'Hospital law, due to the conformal flat requirement, we assume the limit of the coefficient term is equal to 1 . While $\alpha$ is selected as

$$
\alpha \sim \frac{r_{H}\left[\left(r_{H}^{2}+a^{2}\right)\left(2 \dot{r}_{H}-1\right)-\dot{r}_{H} a^{2} \sin ^{2} \theta-2 \Delta_{r_{H}}\right]}{r_{H}\left(2 r_{H}-2 M-4 r_{H} \dot{r}_{H}\right)+2\left[\dot{r}_{H} a^{2} \sin ^{2} \theta-\dot{r}_{H}\left(r_{H}^{2}+a^{2}\right)+r_{H}^{\prime 2}\right]} .
$$


What's worth noting is that, near the even event horizon, $v$ goes to $v_{0}$ and $\theta$ goes to $\theta_{0}[26]$, this becomes

$$
\alpha=\left.\frac{r_{H}\left[\left(r_{H}^{2}+a^{2}\right)\left(2 \dot{r}_{H}-1\right)-\dot{r}_{H} a^{2} \sin ^{2} \theta-2 \Delta_{r_{H}}\right]}{r_{H}\left(2 r_{H}-2 M-4 r_{H} \dot{r}_{H}\right)+2\left[\dot{r}_{H} a^{2} \sin ^{2} \theta-\dot{r}_{H}\left(r_{H}^{2}+a^{2}\right)+r_{H}^{\prime 2}\right]}\right|_{\substack{\nu=\nu_{0} \\ \theta=\theta_{0}}},
$$

both right-hand side and left-hand side in Eq. (22) are constants. From the above expression, it is know that $\alpha$ is not a general constant but a special value corresponds on $v_{0}$ and $\theta_{0}$, so, $\alpha$ should be expressed as $\alpha\left(v_{0}, \theta_{0}\right)$. The physical significance of $\alpha\left(v_{0}, \theta_{0}\right)$ will be discussed later. Near the event horizon Eq. (18) becomes to [24]

$$
\left(\frac{\partial S}{\partial r_{*}}\right)^{2}+A \frac{\partial S}{\partial r_{*}}+2 \frac{\partial S}{\partial r_{*}} \frac{\partial S}{\partial \nu_{*}}+B P_{\theta} \frac{\partial S}{\partial r_{*}}=0
$$

where the limit of $A$ and $B$ are

$$
\begin{gathered}
\left.A\right|_{r \rightarrow r_{H}}=\tilde{A}=\left.\frac{2\left[e Q r_{H}-a\left(1-\dot{r}_{H}\right) j\right]}{\left(r_{H}^{2}+a^{2}\right)-\dot{r}_{H} a^{2} \sin ^{2} \theta}\right|_{\substack{\nu=\nu_{0} \\
\theta=\theta_{0}}}, \\
\left.B\right|_{r \rightarrow r_{H}}=\tilde{B}=\left.2 \frac{r_{H}^{\prime}}{\left(r_{H}^{2}+a^{2}\right)-\dot{r}_{H} a^{2} \sin ^{2} \theta}\right|_{\substack{\nu=\nu_{0} \\
\theta=\theta_{0}}} .
\end{gathered}
$$

Simplified the Eq. (23)

$$
\left(\frac{\partial S}{\partial r_{*}}\right)^{2}+2 \frac{\partial S}{\partial r_{*}}\left(\frac{\partial S}{\partial \nu_{*}}+\omega_{0}\right)=0
$$

Here, we let $\tilde{A}+\tilde{B} P_{\theta_{0}}=\omega_{0}$, where $\omega_{0}$ is a term includes electric potential term. Following the method in ref. [25], we need to prove a conclusion which defined as

$$
\partial S / \partial v_{*}=-\omega
$$

where $\omega$ is the energy of particle, using the function $\rho=C e^{\frac{i S}{\hbar}}$ and

$$
\frac{\partial^{2} \rho}{\partial r_{*}^{2}}+2\left(\frac{\partial}{\partial \nu_{*}}+\frac{i \omega_{0}}{\hbar}\right) \frac{\partial \rho}{\partial r_{*}}=0
$$

It is easy to find that under semi-classical theory both the Eq. (28) and Eq. (26) are equivalent. The result of Eq. (27) gives

$$
\begin{gathered}
\rho_{\text {in }}=C e^{-\frac{i \omega v_{*}}{\hbar}}, \\
\rho_{\text {out }}=C e^{-\frac{i \omega v_{*}-2 i\left(\omega+\omega_{0}\right) r_{*}}{\hbar}} .
\end{gathered}
$$

Now, the formula $\partial S / \partial v_{*}=-\omega$ was proved to be true above. Obviously, it is always established near the event horizon. Substituting Eq. (27) into Eq. (26), the Eq. (26) is reduced to

$$
\frac{\partial S}{\partial r}=\left[1+\frac{\alpha}{r-r_{H}}\right] \frac{\partial S}{\partial r_{*}}=\frac{\left[\left(r-r_{H}\right)+\alpha\right]\left[\left(\omega-\omega_{0}\right) \pm\left(\omega-\omega_{0}\right)\right]}{\left(r-r_{H}\right)},
$$


Here is a singular pole when $r=r_{H}$, after integral around the singular pole, the result is

$$
S=\int \frac{\left[\left(r-r_{H}\right)+\alpha\right]\left[\left(\omega-\omega_{0}\right) \pm\left(\omega-\omega_{0}\right)\right]}{r-r_{H}} \mathrm{~d} r=i \pi \alpha\left[\left(\omega-\omega_{0}\right) \pm\left(\omega-\omega_{0}\right)\right],
$$

where $+(-)$ mean the out going (incoming) solutions. In order to obtains the final tunneling rate, both going solution and incoming solution have be take into account. Therefore, the contribution of imaginary of total action is $[24,25]$

$$
\operatorname{Im} W=\operatorname{Im} S=\operatorname{Im} S_{+}-\operatorname{Im} S_{-}=2 \alpha \pi\left(\omega-\omega_{0}\right) .
$$

So the final expression of tunneling rate is

$$
\Gamma=\Gamma_{\text {emission }} / \Gamma_{\text {absorption }}=e^{-2 \operatorname{Im} W}=e^{-4 \alpha \pi\left(\omega-\omega_{0}\right)},
$$

The physical significance of constant $\alpha\left(v_{0}, \theta_{0}\right)$ is being discussed. Contrast formula $\Gamma=$ $\exp (-\beta E)$ with Eq. (34), where $\beta$ is inverse temperature on the horizon, and the $E$ is the energy of tunneling particle, it is clear to know that the Hawking temperature is depends constant $\alpha\left(v_{0}, \theta_{0}\right)$. Meanwhile, the Hawking temperature is also depends on surface gravity of black hole. This implies that $\alpha\left(v_{0}, \theta_{0}\right)$ under the new type of general tortoise coordination transformation is related to the surface gravity. Furthermore, when let $r_{H}^{\prime}=\dot{r}_{H}=a=Q=0$ and $r_{H}=2 M$ in Eq. (22) the formula becomes to $\alpha=2 M=\frac{1}{2 \kappa_{S}}$, where $\kappa_{S}=\frac{1}{4 M}$ is the surface gravity of Schwarzschild black hole. In another word, the result reduce to the most basic black hole. Know therefore, the relationship between $\alpha\left(v_{0}, \theta_{0}\right)$ and the surface gravity of non-stationary Kerr-Newman is $\alpha\left(v_{0}, \theta_{0}\right)=\frac{1}{2} \kappa_{K-N}$, where the form of $\kappa_{K-N}$ is given by

$$
\kappa_{K-N}=\left.\frac{r_{H}\left(r_{H}-M-2 r_{H} \dot{r}_{H}\right)+\left[\dot{r}_{H} a^{2} \sin ^{2} \theta-\dot{r}_{H}\left(r_{H}^{2}+a^{2}\right)+r_{H}^{\prime 2}\right]}{r_{H}\left[\left(r_{H}^{2}+a^{2}\right)\left(2 \dot{r}_{H}+1\right)-\dot{r}_{H} a^{2} \sin ^{2} \theta-2 \Delta_{r_{H}}\right]}\right|_{\substack{\nu=\nu_{0} \\ \theta=\theta_{0}}} .
$$

Similarly, employing to the expression of tunneling rate $\Gamma_{S}=e^{-\frac{2}{\kappa_{S}} \pi \omega}$ and Hawking temperature of Schwarzschild black hole $T_{H(S)}=\frac{\kappa_{S}}{2 \pi}$ at the point $v=v_{0}, \theta=\theta_{0}$ Eq. (34) means the tunneling rate of non-stationary Kerr-Newman black hole and the Hawking temperature of non-stationary Kerr-Newman black hole is

$$
\begin{aligned}
T_{H(K-N)} & =\frac{\kappa_{K-N}}{2 \pi}=\frac{1}{4 \pi \alpha\left(v_{0}, \theta_{0}\right)} \\
& =\left.\frac{r_{H}\left(r_{H}-M-2 r_{H} \dot{r}_{H}\right)+\left[\dot{r}_{H} a^{2} \sin ^{2} \theta-\dot{r}_{H}\left(r_{H}^{2}+a^{2}\right)+r^{\prime 2}{ }_{H}\right]}{2 \pi r_{H}\left[\left(r_{H}^{2}+a^{2}\right)\left(2 \dot{r}_{H}+1\right)-\dot{r}_{H} a^{2} \sin ^{2} \theta-2 \Delta_{r_{H}}\right]}\right|_{\substack{\nu=v_{0} \\
\theta=\theta_{0}}} .
\end{aligned}
$$

According to the method of Sannan, the radiation spectrum of Schwarzschild black hole is $N_{\omega(S)}=\frac{1}{e^{\omega / K_{B}{ }^{T} H(S)} \pm 1}$. So, the radiation spectrum of non-stationary Kerr-Newman can be written as

$$
N_{\omega(K-N)}=\frac{1}{e^{\omega-\omega_{0} / K_{B} T H(K-N)} \pm 1},
$$

where $K_{B}$ is Boltzmann constant. The + symbol stands for fermion, - symbol means bosons. 


\section{Discussion and Conclusion}

Here introducing the previous general tortoise coordination transformation as

$$
r_{*}=r+\frac{1}{2 \kappa\left(v_{0}, \theta_{0}\right)} \ln \left[r-r_{H}(v, \theta)\right], \quad v_{*}=v-v_{0}, \theta_{*}=\theta-\theta_{0} .
$$

Performing Damour-Ruffini method, the surface gravity becomes

$$
\kappa_{K-N}^{\prime}=\frac{r_{H}\left(1-2 \dot{r}_{H}\right)-M}{\left(r_{H}^{2}+a^{2}-\dot{r}_{H} a^{2} \sin ^{2} \theta_{0}\right)\left(1-2 \dot{r}_{H}\right)+2 r_{H}^{\prime 2}},
$$

the tunneling rate as

$$
\Gamma^{\prime}=e^{-2 \frac{\pi}{\kappa}\left(\omega-\omega_{0}\right)},
$$

and the Hawking temperature as [26]

$$
T_{H}=\frac{r_{H}\left(1-2 \dot{r}_{H}\right)-M}{2 \pi\left[\left(r_{H}^{2}+a^{2}-\dot{r}_{H} a^{2} \sin ^{2} \theta_{0}\right)\left(1-2 \dot{r}_{H}\right)+2 r^{\prime 2}\right]},
$$

the radiation spectrum is

$$
N_{\omega}^{\prime}=\frac{1}{e^{\left(\omega-\omega_{0}\right) / K_{B} T^{\prime}} \pm 1} .
$$

Now, we compare the results under the two types of general tortoise coordinate transformations. Firstly, to take notice of surface gravity $\kappa_{K-N}$ in Eqs. (35) and $\kappa_{K-N}^{\prime}$ in Eqs. (39), it is clear that there is certain difference between $\kappa_{K-N}$ and $\kappa_{K-N}^{\prime}$, which indicated the result we got include more physics information. Furthermore, it is directly led the tunneling rates, Hawking temperatures and radiation spectrums are different under the two types of general tortoise coordinate transformations.

In this paper, the quantum tunneling of the non-stationary Kerr-Newman black hole are obtained via a new type of general tortoise coordinate transformation. Using WKB approximation, the Hamilton-Jacobi equation is derived from Klein-Gordon equation, then we obtained the surface gravity, tunneling rate, Hawking temperature and radiation spectrum of non-stationary Kerr-Newman black hole, when neglecting angular momentum $a$, charge $Q$, parameter $r_{H}^{\prime}, \dot{r}_{H}$ and let $r_{H}=2 M$, it is found that surface gravity is reduced to the situation of Schwarzschild black hole which give full proof means our calculation is correct. Next, by contrast, we found that tunneling rates, Hawking temperatures and radiation spectrums are different under the two types of general tortoise coordinate transformations. According to the dimensional analysis, it is more reasonable and more physically significant to employ the new coordinate system. Therefore, the results we got were more accurate. Remarkably, in the early part of this article we didn't indicated the physical significance of $\alpha\left(v_{0}, \theta_{0}\right)$ as previous work until it is be contrasted with the surface gravity of Schwarzschild black hole, so we think it is more general and conforms with logical. In a word, Eq. (12) should express as $r_{*}=r+\alpha\left(v_{0}, \theta_{0}\right) \ln \left[r-r_{H}(\nu, \theta) / r_{H}(\nu, \theta)\right]$.

It is noteworthy that for the axis symmetric black holes, when $v$ and $\theta$ under the general tortoise coordination transformation are arbitrarily values $r_{*}=r+\alpha(v, \theta) \ln \left[\frac{r-r_{H}(v, \theta)}{r_{H}(v, \theta)}\right]$. This work will have a promising influence on the study of radiation property of black holes. The research results will be reported in our further articles.

Acknowledgements This work is supported by the Natural Science Foundation of China with Grant Nos. 11178018. 
Open Access This article is distributed under the terms of the Creative Commons Attribution License which permits any use, distribution, and reproduction in any medium, provided the original author(s) and the source are credited.

\section{References}

1. Hawking, S.W.: Particle creation by black holes. Commun. Math. Phys. 43, 199-220 (1975)

2. Damour, T., Runi, R.: Black-hole evaporation in the Klein-Sauter-Heisenberg-Euler formalism. Phys. Rev. D 14, 332 (1976)

3. Zhang, J.-Y., Zhao, Z.: Hawking radiation of Dirac particles in a nonuniformly rectilinearly accelerating black hole with electric and magnetic charge. Acta Phys. Sin. 45(8), 2098-2110 (2003) (in Chinese)

4. Jiang, Q.-Q., Wu, S.-Q., Cai, X.: Hawking radiation as tunneling from the Kerr and Kerr-Newman black holes. Phys. Rev. D 73, 064003 (2006). arXiv:hep-th/0512351

5. Chen, D., Yang, H., Zu, X.: Hawking radiation of black holes in the $z=4$ Horava-Lifshitz gravity. Phys. Lett. B 681, 463-468 (2009)

6. Parikh, M.K., Wilczek, F.: Hawking radiation as tunneling. Phys. Rev. Lett. 85, 5042 (2000). arXiv:hep-th/9907001

7. Banerjee, R., Majhi, B.R.: Quantum tunneling and back reaction. Phys. Lett. B 662, 62 (2008). arXiv:0801.0200 [hep-th]

8. Wu, S.-Q., Jiang, Q.-Q.: Hawking radiation of charged particles as tunneling from higher dimensional Reissner-Nordstrom-de Sitter black holes. J. High Energy Phys. 0603, 079 (2006). arXiv:hep-th/0602033

9. Jiang, Q.-Q., Wu, S.-Q.: Hawking radiation of charged particles as tunneling from Reissner-Nordstromde Sitter black holes with a global monopole. Phys. Lett. B 635, 151-155 (2006). arXiv:hep-th/0511123

10. Jiang, Q.-Q.: Dirac particles' tunnelling from black rings. Phys. Rev. D 78, 044009 (2008). arXiv:0807.1358

11. Jiang, Q.-Q., Wu, S.-Q., Cai, X.: Hawking radiation from $(2+1)$-dimensional BTZ black holes. Phys. Lett. B 651, 58-64 (2007). arXiv:hep-th/0701048

12. Srinivasan, K., Padmanabhan, T.: Particle production and complex path analysis. Phys. Rev. D, Part. Fields 60, 24007 (1999)

13. Chen, D.Y., Jiang, Q.Q., Zu, X.T.: Fermions tunnelling from the charged dilatonic black hole. Class. Quantum Gravity 25, 205022 (2008)

14. Chen, D.Y., Jiang, Q.Q., Zu, X.T.: Hawking radiation of Dirac particles via tunneling from rotating black holes in de sitter spaces. Phys. Lett. B 665(2-3), 106-110 (2008)

15. Li, R., Ren, J.R.: Dirac particles tunneling from BTZ black hole. Phys. Lett. B 661, 370-372 (2008)

16. Lin, K., Yang, S.Z.: Fermions tunnelling of a new form Finslerian black hole. Chin. Phys. Lett. 26, 010401 (2009)

17. Lin, K., Yang, S.Z.: Fermions tunneling from the higher-dimensional black hole. Phys. Rev. D, Part. Fields 79(6), 064035 (2009)

18. Lin, K., Yang, S.Z.: Fermions tunneling of higher-dimensional Kerr anti-de-sitter black hole with one rotational parameter. Phys. Lett. B 674(2), 127-130 (2009)

19. Feng, Z.-W., Jiang, Q.-Q., Li, G.-P., Deng, J.: General tortoise coordination transformation and radiation mechanism of dynamical Kerr-Newman black hole. J. Sichuan Univ. Nat. Sci. Ed. 49, 157-162 (2012) (in Chinese)

20. Gonzalez, C., Herrera, L., Jimenez, J.: J. Math. Phys. 20, 837 (1979)

21. Jing, J.L., Wang, Y.J.: Int. J. Theor. Phys. 35, 1481 (1996)

22. Carmeli, M., Kaye, M.: Ann. Phys. 103, 97 (1977)

23. Carmeli, M.: Classical Fields: General Relativity and Gauge Theory. Wiley, New York (1982)

24. Zheng, Y.S., Kai, L.: Sci. China Ser. G 40(4), 507-512 (2010) (in Chinese)

25. Lin, K., Yang, S.-Z.: Fermions tunneling form non-stationary Dilaton-Maxwell black hole via general tortoise coordinate transformation. Chin. Phys. Lett. 26, 100401 (2009)

26. Wu, S.Q., Cai, X.: Hawking radiation of a non-stationary Kerr-Newman black hole: spin-rotation coupling effect. Gen. Relativ. Gravit. 34, 605-617 (2002). arXiv:gr-qc/0111044v1 